\title{
The Schwarz-Pick lemma for slice regular functions
}

\author{
Cinzia Bisi* \\ Università degli Studi di Ferrara \\ Dipartimento di Matematica e Informatica \\ Via Machiavelli 35, 44121 Ferrara, Italy \\ cinzia.bisi@unife.it \\ Caterina Stoppato* $\dagger$ \\ Università degli Studi di Milano \\ Dipartimento di Matematica "F. Enriques" \\ Via Saldini 50, 20133 Milano, Italy \\ caterina.stoppato@unimi.it
}

\begin{abstract}
The celebrated Schwarz-Pick lemma for the complex unit disk is the basis for the study of hyperbolic geometry in one and in several complex variables. In the present paper, we turn our attention to the quaternionic unit ball $\mathbb{B}$. We prove a version of the Schwarz-Pick lemma for self-maps of $\mathbb{B}$ that are slice regular, according to the definition of Gentili and Struppa. The lemma has interesting applications in the fixed-point case, and it generalizes to the case of vanishing higher order derivatives.
\end{abstract}

\section{Introduction}

In the complex case, holomorphy plays a crucial role in the study of the intrinsic geometry of the unit disc $\Delta=\{z \in \mathbb{C}:|z|<1\}$ thanks to the Schwarz-Pick lemma [17, 18.

Theorem 1.1. Let $f: \Delta \rightarrow \Delta$ be a holomorphic function and let $z_{0} \in \Delta$. Then

$$
\left|\frac{f(z)-f\left(z_{0}\right)}{1-\overline{f\left(z_{0}\right)} f(z)}\right| \leq\left|\frac{z-z_{0}}{1-\bar{z}_{0} z}\right|,
$$

*Partially supported by GNSAGA of the INdAM and by FIRB "Geometria Differenziale Complessa e Dinamica Olomorfa".

${ }^{\dagger}$ Partially supported by FSE and by Regione Lombardia. 
for all $z \in \Delta$ and

$$
\frac{\left|f^{\prime}\left(z_{0}\right)\right|}{1-\left|f\left(z_{0}\right)\right|^{2}} \leq \frac{1}{1-\left|z_{0}\right|^{2}}
$$

All inequalities are strict for $z \neq z_{0}$, unless $f$ is a Möbius transformation of $\Delta$.

Some well-known consequences concern the rigidity of holomorphic self-maps of $\Delta$. For instance:

Corollary 1.2. A holomorphic $f: \Delta \rightarrow \Delta$ having more than one fixed point in $\Delta$ must be the identity.

Furthermore, for any holomorphic $f: \Delta \rightarrow \Delta$ such that $f\left(z_{0}\right)=z_{1}$ for fixed $z_{0}, z_{1} \in \Delta$, the modulus $\left|f^{\prime}\left(z_{0}\right)\right|$ cannot exceed $\frac{1-\left|z_{1}\right|^{2}}{1-\left|z_{0}\right|^{2}}$ and it reaches this value if and only if it is a Möbius transformation, 25]. This implies the following special case of the Cartan-Carathéodory theorem.

Theorem 1.3. Let $f$ be a holomorphic self-map of $\Delta$. If $z_{0}$ is a fixed point of $f$ and $f^{\prime}\left(z_{0}\right)=1$ then $f$ coincides with the identity function.

These are the bases for the study of hyperbolic geometry in one and in several complex variables. We refer to [1, 25] for the foundations of this beautiful theory.

Versions of the Schwarz lemma have been proven for the open unit ball

$$
\mathbb{B}=\{q \in \mathbb{H}|| q \mid<1\}
$$

of the real space of quaternions $\mathbb{H}$. Within the theory of regular quaternionic functions introduced by Fueter in 7, 8, which has long been the most successful analog of holomorphy over the quaternions, the article [26 presents a version of the Schwarz lemma for functions $\mathbb{H} \backslash \overline{\mathbb{B}} \rightarrow \mathbb{B}$ that map $\infty$ to 0 and that are Fueter-regular, i.e., that lie in the kernel of $\frac{\partial}{\partial x_{0}}+i \frac{\partial}{\partial x_{1}}+j \frac{\partial}{\partial x_{2}}+k \frac{\partial}{\partial x_{3}}$. (More generally, the analog of the Schwarz lemma presented in [26] is concerned with functions over the Clifford Algebras $C l(0, m))$. See [5, 23] for the foundations of Fueter's theory and of its generalization to the Clifford setting.

Another theory of quaternionic functions, introduced in [11, 12, is based on a different notion of regularity.

Definition 1.4. Let $\Omega$ be a domain in $\mathbb{H}$ and let $f: \Omega \rightarrow \mathbb{H}$ be a function. For all $I \in \mathbb{S}=\left\{q \in \mathbb{H} \mid q^{2}=-1\right\}$, let us denote $L_{I}=\mathbb{R}+I \mathbb{R}, \Omega_{I}=\Omega \cap L_{I}$ and $f_{I}=f_{\left.\right|_{\Omega_{I}}}$. The function $f$ is called (Cullen or) slice regular if, for all $I \in \mathbb{S}$, the restriction $f_{I}$ is real differentiable and the function $\bar{\partial}_{I} f: \Omega_{I} \rightarrow \mathbb{H}$ defined by

$$
\bar{\partial}_{I} f(x+I y)=\frac{1}{2}\left(\frac{\partial}{\partial x}+I \frac{\partial}{\partial y}\right) f_{I}(x+I y)
$$

vanishes identically.

The same articles introduce the Cullen derivative $\partial_{c} f$ of a slice regular function $f$ as

$$
\partial_{c} f(x+I y)=\frac{1}{2}\left(\frac{\partial}{\partial x}-I \frac{\partial}{\partial y}\right) f(x+I y)
$$

for $I \in \mathbb{S}, x, y \in \mathbb{R}$, and they present an analog of the Schwarz lemma. 
Theorem 1.5. Let $f: \mathbb{B} \rightarrow \mathbb{B}$ be a slice regular function. If $f(0)=0$ then

$$
|f(q)| \leq|q|
$$

for all $q \in \mathbb{B}$ and

$$
\left|\partial_{c} f(0)\right| \leq 1 .
$$

Both inequalities are strict (except at $q=0$ ) unless $f(q)=q u$ for some $u \in$ $\partial \mathbb{B}=\{q \in \mathbb{H}|| q \mid=1\}$.

We are presently interested in recovering the full Schwarz-Pick lemma for $\mathbb{B}$. It is known in literature that the set $\mathbb{M}$ of (classical) Möbius transformations of $\mathbb{B}$,

$$
\mathbb{M}=\left\{g(q)=v\left(q-q_{0}\right)\left(1-\bar{q}_{0} q\right)^{-1} u: u, v \in \partial \mathbb{B}, q_{0} \in \mathbb{B}\right\},
$$

is a group with respect to the composition operation and that it is isomorphic to $S p(1,1) /\{ \pm \operatorname{Id}\}$. We recall that

$$
S p(1,1)=\left\{C \in G L(2, \mathbb{H}) \mid \bar{C}^{t} H C=H\right\} \leq S L(2, \mathbb{H}),
$$

where $H=\left[\begin{array}{cc}1 & 0 \\ 0 & -1\end{array}\right], G L(2, \mathbb{H})$ denotes the group of $2 \times 2$ invertible quaternionic matrices, and $S L(2, \mathbb{H})$ denotes the subgroup of those such matrices which have unit Dieudonné determinant (for details, see [3] and references therein). Among the works that treat this matter, even in the more general context of Clifford Algebras, let us mention [2, 15, 24].

The group $\mathbb{M}$, and more in general the group of classical linear fractional transformations $q \mapsto(a q+b)(c q+d)^{-1}$, is not included in Fueter's class. The identity function and the rotations $q \mapsto v q$ and $q \mapsto q u$ with $u, v \in \partial \mathbb{B}$ are examples of classical Möbius transformations that are not Fueter-regular. Thanks to a result of [23], and following [16], one can associate to each transformation $g(q)=v\left(q-q_{0}\right)\left(1-\bar{q}_{0} q\right)^{-1} u$ in $\mathbb{M}$ the Fueter-regular function

$$
G(q)=\frac{\left(1-\bar{q}_{0} q\right)^{-1}}{\left|1-\bar{q}_{0} q\right|^{2}} u \gamma(g(q)),
$$

where $\gamma$ is the Fueter-regular function

$$
\gamma\left(x_{0}+i x_{1}+j x_{2}+k x_{3}\right)=x_{0}+i x_{1}+j x_{2}-k x_{3} .
$$

However, the function $G$ is not, in general, a self-map of $\mathbb{B}$. The variant of the Fueter class considered in [16], defined as the kernel of $\frac{\partial}{\partial x_{0}}+i \frac{\partial}{\partial x_{1}}+j \frac{\partial}{\partial x_{2}}-k \frac{\partial}{\partial x_{3}}$, includes the rotations $q \mapsto v q u$ for all $u \in \partial \mathbb{B}$ and for every $v \in \partial \mathbb{B}$ that is reduced, i.e., whose component along $k$ vanishes. However, the treatment of the rest of the classical Möbius transformations encounters the same kind of difficulties as in Fueter's case.

On the other hand, the class of slice regular functions includes the transformations $q \mapsto\left(q-q_{0}\right)\left(1-q_{0} q\right)^{-1} u$ for $u \in \partial \mathbb{B}$ and $q_{0}$ in the real interval $(-1,1)$. It does not contain the whole group $\mathbb{M}$, but [21] introduced the new 
class of (slice) regular Möbius transformations of $\mathbb{B}$, which are nicely related to the classical ones. They are presented in detail in section 2, which also illustrates several operations that preserve slice regularity: the multiplication $f(q) * g(q)$ of $f(q)$ and $g(q)$, with respect to which every $g \not \equiv 0$ admits an inverse $g(q)^{-*}$; the conjugation $f^{c}(q)$; and the symmetrization $f^{s}(q)=f(q) * f^{c}(q)$.

In section 3. we prove a quaternionic analog of the Schwarz-Pick lemma, which discloses the possibility of using slice regular functions in the study of the intrinsic geometry of $\mathbb{B}$. Before stating our main result, let us recall the basic notions concerning the real differential of a slice regular function. At a real point $x_{0}$, it acts by right multiplication by the Cullen derivative $\partial_{c} f\left(x_{0}\right)$, while at a point $q_{0}=x_{0}+I y_{0} \in L_{I}$ with $I \in \mathbb{S}, x_{0}, y_{0} \in \mathbb{R}$ and $y_{0} \neq 0$ it has been thus characterized in 22]: if we split the tangent space $T_{q_{0}} \Omega \cong \mathbb{H}=\mathbb{R}^{4}$ as $L_{I} \oplus L_{I}^{\perp}$ (with respect to the standard scalar product), then the differential of $f$ at $q_{0}$ acts on $L_{I}$ by right multiplication by $\partial_{c} f\left(q_{0}\right)$; on $L_{I}^{\perp}$, it acts by right multiplication by the spherical derivative

$$
\partial_{s} f\left(q_{0}\right)=\left(2 \operatorname{Im}\left(q_{0}\right)\right)^{-1}\left(f\left(q_{0}\right)-f\left(\bar{q}_{0}\right)\right)
$$

defined in [14]. We recall that the Cullen derivative is a slice regular function, while the spherical derivative is slice regular only when it is constant. The quaternions $\partial_{c} f\left(q_{0}\right)$ and $\partial_{s} f\left(q_{0}\right)$ can be computed as the values at $q_{0}$ and $\bar{q}_{0}$ of a unique slice regular function, which we may call the differential quotient of $f$ at $q_{0}$ :

Remark 1.6. Let $f$ be a slice regular function on $B(0, R)=\{q \in \mathbb{H}|| q \mid<R\}$. If, for all $q_{0} \in \Omega$, we denote as

$$
R_{q_{0}} f(q)=\left(q-q_{0}\right)^{-*} *\left(f(q)-f\left(q_{0}\right)\right)
$$

then $\partial_{c} f\left(q_{0}\right)=R_{q_{0}} f\left(q_{0}\right)$ and $\partial_{s} f\left(q_{0}\right)=R_{q_{0}} f\left(\bar{q}_{0}\right)$.

We are now in a position to state the main result of the present article.

Theorem 1.7 (Schwarz-Pick lemma). Let $f: \mathbb{B} \rightarrow \mathbb{B}$ be a regular function and let $q_{0} \in \mathbb{B}$. Then in $\mathbb{B}$

$$
\begin{aligned}
\left|\left(f(q)-f\left(q_{0}\right)\right) *\left(1-\overline{f\left(q_{0}\right)} * f(q)\right)^{-*}\right| & \leq\left|\left(q-q_{0}\right) *\left(1-\bar{q}_{0} * q\right)^{-*}\right| \\
\left|R_{q_{0}} f(q) *\left(1-\overline{f\left(q_{0}\right)} * f(q)\right)^{-*}\right| & \leq\left|\left(1-\bar{q}_{0} * q\right)^{-*}\right|
\end{aligned}
$$

Moreover,

$$
\begin{aligned}
\left|\partial_{c} f *\left(1-\overline{f\left(q_{0}\right)} * f(q)\right)^{-*}\right|_{q_{0}} & \leq \frac{1}{1-\left|q_{0}\right|^{2}} \\
\frac{\left|\partial_{s} f\left(q_{0}\right)\right|}{\left|1-f^{s}\left(q_{0}\right)\right|} & \leq \frac{1}{\left|1-{\overline{q_{0}}}^{2}\right|}
\end{aligned}
$$

If $f$ is a slice regular Möbius transformation of $\mathbb{B}$ then equality holds in the previous formulas. Else, all the aforementioned inequalities are strict (except for the first one at $q_{0}$, which reduces to $0 \leq 0$ ). 
We conclude section 3 computing a point $\tilde{q}_{0}$ with $\operatorname{Re}\left(\tilde{q}_{0}\right)=\operatorname{Re}\left(q_{0}\right)$ and $\left|\operatorname{Im}\left(\tilde{q}_{0}\right)\right|=\left|\operatorname{Im}\left(q_{0}\right)\right|$ such that

$$
\left|\partial_{c} f *\left(1-\overline{f\left(q_{0}\right)} * f(q)\right)^{-*}\right|_{q_{0}}=\frac{\left|\partial_{c} f\left(q_{0}\right)\right|}{\left|1-\overline{f\left(q_{0}\right)} f\left(\tilde{q}_{0}\right)\right|} .
$$

As an application of the main theorem, in section 4 we obtain direct proofs of the quaternionic analogs of the Cartan Rigidity theorems mentioned at the beginning of this introduction. Versions of these results have been proven in [13, and our new approach allows to strengthen their statements.

Theorem 1.8. Let $f: \mathbb{B} \rightarrow \mathbb{B}$ be a slice regular function and suppose $f$ to have a fixed point $q_{0} \in \mathbb{B}$. Then either $f$ is the identity function, or $f$ has no other fixed point in $\mathbb{B}$.

Theorem 1.9. Let $f: \mathbb{B} \rightarrow \mathbb{B}$ be a slice regular function and suppose $f$ to have a fixed point $q_{0} \in \mathbb{B}$. The following facts are equivalent:

(1) $f$ coincides with the identity function;

(2) the real differential of $f$ at $q_{0}$ is the identity;

(3) the Cullen derivative $\partial_{c} f\left(q_{0}\right)$ equals 1 ;

(4) the spherical derivative $\partial_{s} f\left(q_{0}\right)$ equals 1 ;

(5) $R_{q_{0}} f(q)$ equals $\left(1-\bar{q}_{0} * q\right)^{-*} *\left(1-\bar{q}_{0} * f(q)\right)$ at some $q \in \mathbb{B}$.

Finally, in section 5 we generalize our version of the Schwarz-Pick lemma to the case of vanishing higher order derivatives.

\section{Acknowledgements}

The authors wish to thank Graziano Gentili for his enthusiasm for this topic, and for the fruitful discussions that originated this work. They are also grateful to the anonymous referee for her/his precious suggestions.

\section{Regular Möbius transformations of $\mathbb{B}$}

This section surveys the algebraic structure of slice regular functions, and its application to the construction of regular fractional transformations. From now on, we will omit the term 'slice' and refer to these functions as regular, tout court. Since we will be interested only in regular functions on Euclidean balls $B(0, R)$ of radius $R$ centered at 0 , or on the whole space $\mathbb{H}=B(0,+\infty)$, we will follow the presentation of $[9,20$. However, we point out that many of the results we are about to mention have been generalized to a larger class of domains in 6 . 
Theorem 2.1. Fix $R$ with $0<R \leq+\infty$ and let

$$
\mathcal{D}_{R}=\{f: B(0, R) \rightarrow \mathbb{H} \mid f \text { regular }\} .
$$

Then $\mathcal{D}_{R}$ coincides with the set of quaternionic power series $f(q)=\sum_{n \in \mathbb{N}} q^{n} a_{n}$ (with $a_{n} \in \mathbb{H}$ ) converging in $B(0, R)$. Moreover, $\mathcal{D}_{R}$ is an associative real algebra with respect to + and to the regular multiplication $*$ defined on $f(q)=$ $\sum_{n \in \mathbb{N}} q^{n} a_{n}$ and $g(q)=\sum_{n \in \mathbb{N}} q^{n} b_{n}$ by the formula

$$
f * g(q)=\sum_{n \in \mathbb{N}} q^{n} \sum_{k=0}^{n} a_{k} b_{n-k} .
$$

We will also write $f(q) * g(q)$ for $f * g(q)$. In this case, the letter $q$ will always denote the variable. The ring $\mathcal{D}_{R}$ admits a classical ring of quotients

$$
\mathcal{L}_{R}=\left\{f^{-*} * g \mid f, g \in \mathcal{D}_{R}, f \not \equiv 0\right\} .
$$

In order to introduce it, we begin with the following definition.

Definition 2.2. Let $f(q)=\sum_{n \in \mathbb{N}} q^{n} a_{n}$ be a regular function on an open ball $B=B(0, R)$. The regular conjugate of $f, f^{c}: B \rightarrow \mathbb{H}$, is defined as $f^{c}(q)=$ $\sum_{n \in \mathbb{N}} q^{n} \bar{a}_{n}$ and the symmetrization of $f$, as $f^{s}=f * f^{c}=f^{c} * f$.

Notice that $f^{s}(q)=\sum_{n \in \mathbb{N}} q^{n} r_{n}$ with $r_{n}=\sum_{k=0}^{n} a_{k} \bar{a}_{n-k} \in \mathbb{R}$. Moreover, the zero-sets of $f^{c}$ and $f^{s}$ have been fully characterized.

Theorem 2.3. Let $f$ be a regular function on $B=B(0, R)$. For all $x, y \in \mathbb{R}$ with $x+y \mathbb{S} \subseteq B$, the regular conjugate $f^{c}$ has as many zeros as $f$ in $x+y \mathbb{S}$. Moreover, the zero set of the symmetrization $f^{s}$ is the union of all the $x+y \mathbb{S}$ on which $f$ has a zero.

We are now ready for the definition of regular quotient. We denote by

$$
\mathcal{Z}_{h}=\{q \in B \mid h(q)=0\}
$$

the zero-set of a function $h$.

Definition 2.4. Let $f, g: B=B(0, R) \rightarrow \mathbb{H}$ be regular functions. The left regular quotient of $f$ and $g$ is the function $f^{-*} * g$ defined in $B \backslash \mathcal{Z}_{f^{s}}$ by

$$
f^{-*} * g(q)=f^{s}(q)^{-1} f^{c} * g(q) .
$$

Moreover, the regular reciprocal of $f$ is the function $f^{-*}=f^{-*} * 1$.

Left regular quotients proved to be regular in their domains of definition. If we set $\left(f^{-*} * g\right) *\left(h^{-*} * k\right)=\left(f^{s} h^{s}\right)^{-1} f^{c} * g * h^{c} * k$ then $\left(\mathcal{L}_{R},+, *\right)$ is a division algebra over $\mathbb{R}$ and it is the classical ring of quotients of $\left(\mathcal{D}_{R},+, *\right)$ (for this notion, see [19]). In particular, $\mathcal{L}_{R}$ coincides with the set of right regular quotients

$$
g * h^{-*}(q)=h^{s}(q)^{-1} g * h^{c}(q) .
$$


The definition of regular conjugation and symmetrization is extended to $\mathcal{L}_{R}$ setting $\left(f^{-*} * g\right)^{c}=g^{c} *\left(f^{c}\right)^{-*}$ and $\left(f^{-*} * g\right)^{s}(q)=f^{s}(q)^{-1} g^{s}(q)$. Furthermore, the following relation between the left regular quotient $f^{-*} * g(q)$ and the quotient $f(q)^{-1} g(q)$ holds.

Theorem 2.5. Let $f, g$ be regular functions on $B=B(0, R)$. Then

$$
f * g(q)= \begin{cases}0 & \text { if } f(q)=0 \\ f(q) g\left(f(q)^{-1} q f(q)\right) & \text { otherwise }\end{cases}
$$

and setting $T_{f}(q)=f^{c}(q)^{-1} q f^{c}(q)$ for all $q \in B \backslash \mathcal{Z}_{f^{s}}$,

$$
f^{-*} * g(q)=f\left(T_{f}(q)\right)^{-1} g\left(T_{f}(q)\right)
$$

for all $q \in B \backslash \mathcal{Z}_{f^{s}}$. For all $x, y \in \mathbb{R}$ with $x+y \mathbb{S} \subset B \backslash \mathcal{Z}_{f^{s}} \subset B \backslash \mathcal{Z}_{f^{c}}$, the function $T_{f}$ maps $x+y \mathbb{S}$ to itself (in particular $T_{f}(x)=x$ for all $x \in \mathbb{R}$ ). Furthermore, $T_{f}$ is a diffeomorphism from $B \backslash \mathcal{Z}_{f}$ onto itself, with inverse $T_{f^{c}}$.

We point out that, so far, no similar result relating $g * h^{-*}(q)$ to $g(q) h(q)^{-1}$ is known.

This machinery allowed the introduction in 21 of regular analogs of linear fractional transformations. To each $A=\left[\begin{array}{ll}a & c \\ b & d\end{array}\right] \in G L(2, \mathbb{H})$ we can associate the regular fractional transformation

$$
\mathcal{F}_{A}(q)=(q c+d)^{-*} *(q a+b)
$$

By the formula $(q c+d)^{-*} *(q a+b)$ we denote the aforementioned left regular quotient $f^{-*} * g$ of $f(q)=q c+d$ and $g(q)=q a+b$. We denote the $2 \times 2$ identity matrix as Id. The set of regular fractional transformations

$$
\mathfrak{G}=\left\{\mathcal{F}_{A} \mid A \in G L(2, \mathbb{R})\right\}
$$

is not a group, but it is the orbit of the identity function $i d=\mathcal{F}_{\text {Id }}$ with respect to the two actions on $\mathcal{L}_{\infty}$ described in the next theorem.

Theorem 2.6. Choose $R>0$ and consider the ring of quotients of regular quaternionic functions in $B(0, R)$, denoted by $\mathcal{L}_{R}$. Setting

$$
f \cdot A=(f c+d)^{-*} *(f a+b)
$$

for all $f \in \mathcal{L}_{R}$ and for all $A=\left[\begin{array}{ll}a & c \\ b & d\end{array}\right] \in G L(2, \mathbb{H})$, defines a right action of $G L(2, \mathbb{H})$ on $\mathcal{L}_{R}$. A left action of $G L(2, \mathbb{H})$ on $\mathcal{L}_{R}$ is defined setting

$$
A^{t} \cdot f=(a * f+b) *(c * f+d)^{-*} .
$$

The stabilizer of any element of $\mathcal{L}_{R}$ with respect to either action includes the normal subgroup $N=\{t \cdot \mathrm{Id} \mid t \in \mathbb{R} \backslash\{0\}\} \unlhd G L(2, \mathbb{H})$. Both actions are faithful, but not free, when reduced to $\operatorname{PSL}(2, \mathbb{H})=G L(2, \mathbb{H}) / N$. 
For more details, see [4, 21]. The two actions are related as follows.

Proposition 2.7. For all $A \in G L(2, \mathbb{H})$ and for all $f \in \mathcal{L}_{R}$

1. $(f \cdot A)^{c}=\bar{A}^{t} \cdot f^{c}$;

2. if $A$ is Hermitian then $f . A=A^{t} . f$;

3. if $A$ is Hermitian then $(f \cdot A)^{c}=f^{c} \cdot \bar{A}$.

As a consequence, the set $\mathfrak{G}$ of regular fractional transformations is preserved by regular conjugation. For the proofs of these properties, we refer the reader to [4].

In the present paper, we are specifically interested in those regular fractional transformations that map the open quaternionic unit ball $\mathbb{B}$ onto itself, called regular Möbius transformations of $\mathbb{B}$, whose class we denote as

$$
\mathfrak{M}=\{f \in \mathfrak{G} \mid f(\mathbb{B})=\mathbb{B}\} .
$$

More generally, we will concern ourselves with the class

$$
\mathfrak{R e g}(\mathbb{B}, \mathbb{B})=\{f: \mathbb{B} \rightarrow \mathbb{B} \mid f \text { is regular }\}
$$

of regular self-maps of $\mathbb{B}$. It was proven in 21 that a function $f \in \mathfrak{R e g}(\mathbb{B}, \mathbb{B})$ is a regular Möbius transformation if, and only if, it is bijective. Furthermore, the next property was proven in [4, 21].

Theorem 2.8. A function $f: \mathbb{B} \rightarrow \mathbb{H}$ is a regular Möbius transformation of $\mathbb{B}$ if and only if there exist (unique) $u \in \partial \mathbb{B}, a \in \mathbb{B}$ such that

$$
f(q)=\left(q-q_{0}\right) *\left(1-\bar{q}_{0} * q\right)^{-*} u=\left(1-q \bar{q}_{0}\right)^{-*} *\left(q-q_{0}\right) u
$$

In other words, $\mathfrak{M}$ is the orbit of the identity function under the left and right actions of $S p(1,1)$.

We point out that, by definition, $\bar{q}_{0} * q=q \bar{q}_{0}$. Finally, let us recall a result that will prove useful in the sequel (see proposition 3.3 of [4).

Proposition 2.9. If $f \in \mathfrak{R e g}(\mathbb{B}, \mathbb{B})$ then for all $a \in \mathbb{B}$

$$
(f(q)-a) *(1-\bar{a} * f(q))^{-*}=(1-f(q) \bar{a})^{-*} *(f(q)-a) .
$$

Furthermore, the left and right actions of $S p(1,1)$ and the regular conjugation preserve both $\mathfrak{R e g}(\mathbb{B}, \mathbb{B})$ and $\mathfrak{M}$.

\section{The Schwarz-Pick lemma}

In this section, we shall prove the announced Schwarz-Pick lemma for quaternionic regular functions. In order to obtain it, we begin with a result concerning the special case of a function $f: \mathbb{B} \rightarrow \mathbb{B}$ having a zero. We follow the line of the complex proof, making use of the maximum modulus principle for regular functions proven in 12 . 
Theorem 3.1. Let $f: B(0, R) \rightarrow \mathbb{H}$ be a regular function. If $|f|$ has a relative maximum at $p \in B(0, R)$, then $f$ is constant.

We now turn to the aforementioned result.

Theorem 3.2. If $f: \mathbb{B} \rightarrow \mathbb{B}$ is regular and if $f\left(q_{0}\right)=0$ for some $q_{0} \in \mathbb{B}$, and if

$$
\mathcal{M}_{q_{0}}(q)=\left(q-q_{0}\right) *\left(1-q \bar{q}_{0}\right)^{-*}=\left(1-q \bar{q}_{0}\right)^{-*} *\left(q-q_{0}\right),
$$

then

$$
\left|\mathcal{M}_{q_{0}}^{-*} * f(q)\right| \leq 1
$$

for all $q \in \mathbb{B}$. The inequality is strict, unless $\mathcal{M}_{q_{0}}^{-*} * f(q) \equiv u$ for some $u \in \partial \mathbb{B}$.

Proof. Let us consider $\mathcal{M}_{q_{0}}^{-*}(q)=\left(1-q \bar{q}_{0}\right) *\left(q-q_{0}\right)^{-*}=\left(q-q_{0}\right)^{-*} *\left(1-q \bar{q}_{0}\right)$, which is a regular function on $\mathbb{B}$ minus the 2-sphere $S_{q_{0}}=x_{0}+y_{0} \mathbb{S}$ through $q_{0}$ (that is, minus the zero set of $\left.\left(q-q_{0}\right)^{s}=\left(q-x_{0}\right)^{2}+y_{0}^{2}\right)$. Since $f\left(q_{0}\right)=0$, we have $f(q)=\left(q-q_{0}\right) * R_{q_{0}} f(q)$ where $R_{q_{0}} f: \mathbb{B} \rightarrow \mathbb{H}$ is the differential quotient defined by formula (8). Hence setting

$$
h(q)=\mathcal{M}_{q_{0}}^{-*} * f(q)=\left(1-q \bar{q}_{0}\right) * R_{q_{0}} f(q)
$$

defines a regular function on $\mathbb{B}$. Moreover, by the first part of theorem 2.5

$$
h(q)=\mathcal{M}_{q_{0}}^{-*} * f(q)=\mathcal{M}_{q_{0}}^{-*}(q) f\left(g(q)^{-1} q g(q)\right)
$$

where $g=\mathcal{M}_{q_{0}}^{-*}$. Since $|f|<1$ in $\mathbb{B}$, we conclude that

$$
|h(q)|=\left|\mathcal{M}_{q_{0}}^{-*} * f(q)\right| \leq\left|\mathcal{M}_{q_{0}}^{-*}(q)\right|
$$

away from $S_{q_{0}}$. Applying the second part of theorem 2.5, we notice that for all $q \in \mathbb{B} \backslash S_{q_{0}}$

$$
\mathcal{M}_{q_{0}}^{-*}(q)=\left(T_{l}(q)-q_{0}\right)^{-1}\left(1-T_{l}(q) \bar{q}_{0}\right)=\left[M_{q_{0}}\left(T_{l}(q)\right)\right]^{-1}
$$

where $l(q)=q-q_{0}$, and where $M_{q_{0}}(q)=\left(1-q \bar{q}_{0}\right)^{-1}\left(q-q_{0}\right)$. Now, $M_{q_{0}}$ maps $\mathbb{B}$ onto itself and $\partial \mathbb{B}$ onto itself, and for all $\varepsilon>0$ there exists $r$ with $\left|q_{0}\right|<r<1$ such that

$$
1 \leq\left|M_{q_{0}}(q)\right|^{-1} \leq 1+\varepsilon
$$

for $|q| \geq r$. Hence,

$$
\max _{|q|=r}|h(q)| \leq \max _{|q|=r}\left|\mathcal{M}_{q_{0}}^{-*}(q)\right|=\max _{|q|=r}\left|M_{q_{0}}\left(T_{l}(q)\right)\right|^{-1}=\max _{|w|=r}\left|M_{q_{0}}(w)\right|^{-1} \leq 1+\varepsilon .
$$

Suppose there existed $p \in \mathbb{B}, \delta>0$ such that $|h(p)|=1+\delta$. There would exist $r>|p|$ (with $r>\left|q_{0}\right|$ ) such that $\max _{|q|=r}|h(q)| \leq 1+\delta / 2$ and, by the maximum modulus principle $|h(q)| \leq 1+\delta / 2$ for $|q| \leq r$. We would then have $|h(p)| \leq 1+\delta / 2$, a contradiction with the hypothesis. Hence $\left|\mathcal{M}_{q_{0}}^{-*} * f(q)\right| \leq 1$ for all $q \in \mathbb{B}$.

We conclude observing that, since $\left|\mathcal{M}_{q_{0}}^{-*} * f(q)\right| \leq 1$ for all $q \in \mathbb{B}$, if there exists $\tilde{q} \in \mathbb{B}$ such that $\left|\mathcal{M}_{q_{0}}^{-*} * f(\tilde{q})\right|=1$ then by the maximum modulus principle 3.1. $\mathcal{M}_{q_{0}}^{-*} * f$ must be a constant $u \in \partial \mathbb{B}$. 
In order to reformulate the previous result as an analog of the Schwarz-Pick lemma, we will need some other instruments. The first of them is the following lemma.

Lemma 3.3. Let $f, g, h: B=B(0, R) \rightarrow \mathbb{H}$ be regular functions. If $|f| \leq|g|$ then $|h * f| \leq|h * g|$. Moreover, if $|f|<|g|$ then $|h * f|<|h * g|$ in $B \backslash \mathcal{Z}_{h}$.

Proof. If $|f| \leq|g|$ then for all $q \in B \backslash \mathcal{Z}_{h}$

$$
\left|f\left(h(q)^{-1} q h(q)\right)\right| \leq\left|g\left(h(q)^{-1} q h(q)\right)\right|
$$

so that

$$
|h * f(q)|=|h(q)| \cdot\left|f\left(h(q)^{-1} q h(q)\right)\right| \leq|h(q)| \cdot\left|g\left(h(q)^{-1} q h(q)\right)\right||=| h * g(q) \mid
$$

thanks to theorem 2.5. The reasoning is also valid if all the inequalities are substituted by strict inequalities. Finally, for all $q \in \mathcal{Z}_{h}$ we have $|h * f(q)|=$ $0=|h * g(q)|$.

Secondly, let us compute the differential quotient (and the derivatives) of $\mathcal{M}_{q_{0}}$ at $q_{0}$.

Remark 3.4. In the case of the regular Möbius transformation $\mathcal{M}_{q_{0}}$, clearly $R_{q_{0}} \mathcal{M}_{q_{0}}(q)=\left(1-q \bar{q}_{0}\right)^{-*}$, so that $\partial_{c} f\left(q_{0}\right)=\frac{1}{1-\left|q_{0}\right|^{2}}$ and $\partial_{s} f\left(q_{0}\right)=\frac{1}{1-{\overline{q_{0}}}^{2}}$.

We are now in a position to suitably restate our result.

Corollary 3.5. If $f: \mathbb{B} \rightarrow \mathbb{B}$ is regular, if $q_{0} \in \mathbb{B}$ and if $f\left(q_{0}\right)=0$ then

$$
|f(q)| \leq\left|\mathcal{M}_{q_{0}}(q)\right|
$$

for all $q \in \mathbb{B}$. The inequality is strict at all $q \in \mathbb{B} \backslash\left\{q_{0}\right\}$, unless there exists $u \in$ $\partial \mathbb{B}$ such that $f(q)=\mathcal{M}_{q_{0}}(q) \cdot u$ at all $q \in \mathbb{B}$. Moreover, $\left|R_{q_{0}} f(q)\right| \leq\left|\left(1-q \bar{q}_{0}\right)^{-*}\right|$ in $\mathbb{B}$ and in particular

$$
\begin{gathered}
\left|\partial_{c} f\left(q_{0}\right)\right| \leq \frac{1}{1-\left|q_{0}\right|^{2}} \\
\left|\partial_{s} f\left(q_{0}\right)\right| \leq \frac{1}{\left|1-{\overline{q_{0}}}^{2}\right|} .
\end{gathered}
$$

These inequalities are strict, unless $f(q)=\mathcal{M}_{q_{0}}(q) \cdot u$ for some $u \in \partial \mathbb{B}$.

Proof. By theorem 3.2 .

$$
\left|\mathcal{M}_{q_{0}}^{-*} * f(q)\right| \leq 1
$$

for all $q \in \mathbb{B}$. Since $\mathcal{M}_{q_{0}}^{-*} * f(q)$ and the constant 1 are regular in $\mathbb{B}$, by lemma 3.3

$$
|f| \leq\left|\mathcal{M}_{q_{0}}\right|
$$

in $\mathbb{B}$. According to theorem 3.2 all inequalities above are strict for $q \in \mathbb{B} \backslash\left\{q_{0}\right\}$, unless there exists $u \in \partial \mathbb{B}$ such that $\mathcal{M}_{q_{0}}^{-*} * f(q) \equiv u$, that is, $f(q)=\mathcal{M}_{q_{0}}(q) \cdot u$ for all $q \in \mathbb{B}$. 
The second statement follows from

$$
1 \geq\left|\mathcal{M}_{q_{0}}^{-*} * f(q)\right|=\left|\left(1-q \bar{q}_{0}\right) *\left(q-q_{0}\right)^{-*} * f(q)\right|=\left|\left(1-q \bar{q}_{0}\right) * R_{q_{0}} f(q)\right|
$$

applying lemma 3.3 , since $\left(1-q \bar{q}_{0}\right)^{-*}$ is regular and has no zeros in $\mathbb{B}$.

We shall now generalize the previous corollary to an analog of the SchwarzPick lemma. We will make use of the Leibniz rules for $\partial_{c}, \partial_{s}$, from [10] and [14] respectively. We recall that if $f$ is regular in $B(0, R)$ then

$$
f(q)=v_{s} f(q)+\operatorname{Im}(q) \partial_{s} f(q)
$$

for all $q \in B(0, R)$ where $v_{s} f$ denotes the spherical value $v_{s} f(q)=\frac{f(q)+f(\bar{q})}{2}$ for all $q \in B(0, R)$.

Remark 3.6. If $f: B(0, R) \rightarrow \mathbb{H}$ is a regular function then

$$
\begin{aligned}
& \partial_{c}(f * g)(q)=\partial_{c} f(q) * g(q)+f(q) * \partial_{c} g(q) \\
& \partial_{s}(f * g)(q)=\partial_{s} f(q) \cdot v_{s} g(q)+v_{s} f(q) \cdot \partial_{s} g(q) .
\end{aligned}
$$

Theorem 3.7 (Schwarz-Pick lemma). Let $f: \mathbb{B} \rightarrow \mathbb{B}$ be a regular function and let $q_{0} \in \mathbb{B}$. Then in $\mathbb{B}$

$$
\begin{aligned}
\left|\left(f(q)-f\left(q_{0}\right)\right) *\left(1-\overline{f\left(q_{0}\right)} * f(q)\right)^{-*}\right| & \leq\left|\left(q-q_{0}\right) *\left(1-\bar{q}_{0} * q\right)^{-*}\right| \\
\left|R_{q_{0}} f(q) *\left(1-\overline{f\left(q_{0}\right)} * f(q)\right)^{-*}\right| & \leq\left|\left(1-\bar{q}_{0} * q\right)^{-*}\right|
\end{aligned}
$$

Moreover,

$$
\begin{aligned}
\left|\partial_{c} f *\left(1-\overline{f\left(q_{0}\right)} * f(q)\right)^{-*}\right|_{q_{0}} & \leq \frac{1}{1-\left|q_{0}\right|^{2}} \\
\frac{\left|\partial_{s} f\left(q_{0}\right)\right|}{\left|1-f^{s}\left(q_{0}\right)\right|} & \leq \frac{1}{\left|1-{\overline{q_{0}}}^{2}\right|}
\end{aligned}
$$

If $f$ is a regular Möbius transformation then equality holds in (28), (29) for all $q \in \mathbb{B}$, and in (30), (31). Else, all the aforementioned inequalities are strict (except for (28) at $q_{0}$, which reduces to $0 \leq 0$ ).

Proof. Thanks to proposition 2.9

$$
\tilde{f}(q)=\left(f(q)-f\left(q_{0}\right)\right) *\left(1-\overline{f\left(q_{0}\right)} * f(q)\right)^{-*}
$$

is a regular function $\mathbb{B} \rightarrow \mathbb{B}$. Since $f(q)-f\left(q_{0}\right)$ has a zero at $q_{0}$, by theorem 2.5 the product $\tilde{f}$ has the additional property that $\tilde{f}\left(q_{0}\right)=0$. Inequalities (28) and (29) now follow applying corollary 3.5 to $\tilde{f}$ (taking into account that $\left.\bar{q}_{0} * q=q \bar{q}_{0}\right)$. They are strict unless $\tilde{f}(q)=\mathcal{M}_{q_{0}}(q) \cdot u$ for some $u \in \partial \mathbb{B}$, which is true if and only if $f$ is a regular Möbius transformation of $\mathbb{B}$.

Inequality (30) follows from corollary 3.5 and from the fact that, according to formula (26)

$\partial_{c} \tilde{f}(q)=\partial_{c} f(q) *\left(1-\overline{f\left(q_{0}\right)} * f(q)\right)^{-*}+\left(f(q)-f\left(q_{0}\right)\right) * \partial_{c}\left(1-\overline{f\left(q_{0}\right)} * f(q)\right)^{-*}$, 
where the second term vanishes at $q_{0}$ by theorem 2.5 .

As for (31), it derives again from corollary 3.5 proving that

$$
\partial_{s} \tilde{f}\left(q_{0}\right)=\left[1-\overline{f^{s}\left(q_{0}\right)}\right]^{-1} \partial_{s} f\left(q_{0}\right)
$$

Indeed, setting $g(q)=\left(1-f(q) \overline{f\left(q_{0}\right)}\right)^{-*}$, formula (27) and proposition 2.9 imply

$$
\begin{aligned}
\partial_{s} \tilde{f}\left(q_{0}\right) & =\partial_{s}\left[g(g) *\left(f(q)-f\left(q_{0}\right)\right)\right]_{\left.\right|_{q_{0}}} \\
& =\partial_{s} g\left(q_{0}\right) \cdot v_{s}\left(f(q)-f\left(q_{0}\right)\right)_{\left.\right|_{0}}+v_{s} g\left(q_{0}\right) \cdot \partial_{s}\left(f(q)-f\left(q_{0}\right)\right)_{\left.\right|_{0}} \\
& =-\partial_{s} g\left(q_{0}\right) \cdot \operatorname{Im}\left(q_{0}\right) \cdot \partial_{s} f\left(q_{0}\right)+v_{s} g\left(q_{0}\right) \cdot \partial_{s} f\left(q_{0}\right) \\
& =\overline{\left.\overline{v_{s} g\left(q_{0}\right)}+\operatorname{Im}\left(q_{0}\right) \overline{\partial_{s} g\left(q_{0}\right)}\right]} \cdot \partial_{s} f\left(q_{0}\right) \\
& =\overline{g^{c}\left(q_{0}\right)} \cdot \partial_{s} f\left(q_{0}\right)
\end{aligned}
$$

where we have taken into account that, according to [14, $v_{s} g^{c}(q)=\overline{v_{s} g(q)}$ and $\partial_{s} g^{c}(q)=\overline{\partial_{s} g(q)}$. Thanks to theorem 2.5 $g^{c}(q)=h^{-*}(q)=h\left(T_{h}(q)\right)^{-1}$ where $h(q)=\left(1-f(q) \overline{f\left(q_{0}\right)}\right)^{c}=1-f\left(q_{0}\right) * f^{c}(q)$ and

$$
T_{h}(q)=\left(1-f(q) \overline{f\left(q_{0}\right)}\right)^{-1} q\left(1-f(q) \overline{f\left(q_{0}\right)}\right) .
$$

Since

$$
1-f\left(q_{0}\right) \overline{f\left(q_{0}\right)}=1-\left|f\left(q_{0}\right)\right|^{2}
$$

is real, $T_{h}\left(q_{0}\right)=q_{0}$ and $g^{c}\left(q_{0}\right)=h\left(q_{0}\right)^{-1}$. Furthermore, if $f(q)=\sum_{n \in \mathbb{N}} q^{n} a_{n}$ then

$$
f\left(q_{0}\right) * f^{c}(q)=f\left(q_{0}\right) * \sum_{n \in \mathbb{N}} q^{n} \bar{a}_{n}=\sum_{n \in \mathbb{N}} q^{n} f\left(q_{0}\right) \bar{a}_{n}=\sum_{m, n \in \mathbb{N}} q^{n} q_{0}^{m} a_{m} \bar{a}_{n}
$$

equals $f^{s}(q)=\sum_{k \in \mathbb{N}} q^{k} \sum_{m=0}^{k} a_{m} \bar{a}_{k-m}$ at $q_{0}$. Hence, $h\left(q_{0}\right)=1-f^{s}\left(q_{0}\right)$, and the proof is complete.

According to theorem 2.5

$$
\partial_{c} f *\left(1-\overline{f\left(q_{0}\right)} * f(q)\right)_{\left.\right|_{q_{0}}}^{-*}=\partial_{c} f\left(q_{0}\right)\left(1-\overline{f\left(q_{0}\right)} f\left(\tilde{q}_{0}\right)\right)^{-1}
$$

where $\tilde{q}_{0}=T_{g}\left(\partial_{c} f\left(q_{0}\right)^{-1} q_{0} \partial_{c} f\left(q_{0}\right)\right)$ and $g(q)=1-\overline{f\left(q_{0}\right)} * f(q)$. Hence, inequality (30) can be restated as

$$
\frac{\left|\partial_{c} f\left(q_{0}\right)\right|}{\left|1-\overline{f\left(q_{0}\right)} f\left(\tilde{q}_{0}\right)\right|} \leq \frac{1}{1-\left|q_{0}\right|^{2}}
$$

which closely resembles the complex estimate (2). 


\section{Applications of the Schwarz-Pick lemma}

As an application of our main result 3.7 we study the fixed point case extending the work done in [13. In the proofs, we thoroughly use the following property of the zero set proven in 9] (an immediate consequence of theorem 2.5).

Corollary 4.1. Let $f, g, h$ be regular functions on $B=B(0, R)$. Then $f *$ $g$ vanishes at a point $q_{0}=x_{0}+I y_{0}$ if, and only if, either $f\left(q_{0}\right)=0$ or $f\left(q_{0}\right)^{-1} q_{0} f\left(q_{0}\right)=x_{0}+f\left(q_{0}\right)^{-1} \operatorname{If}\left(q_{0}\right) y_{0}$ is a zero of $g$. As a consequence, if $f, h$ vanish nowhere in $B$ then each $x_{0}+y_{0} \mathbb{S} \subset B$ contains as many zeros of $f * g * h$ as zeros of $g$.

We are now ready for our study. The next result had been proven in [13] in the special case when $q_{0}$ is real, in the interval $(-1,1)$.

Theorem 4.2. Let $f: \mathbb{B} \rightarrow \mathbb{B}$ be a regular function and suppose $f$ to have a fixed point $q_{0} \in \mathbb{B}$. Then either $f$ is the identity function, or $f$ has no other fixed point in $\mathbb{B}$.

Proof. Since $f\left(q_{0}\right)=q_{0}$, the inequality (28) becomes $|\tilde{f}| \leq\left|\mathcal{M}_{q_{0}}\right|$ with

$$
\tilde{f}(q)=\left(f(q)-q_{0}\right) *\left(1-\bar{q}_{0} * f(q)\right)^{-*}=\left(1-f(q) \bar{q}_{0}\right)^{-*} *\left(f(q)-q_{0}\right) .
$$

Let us consider the set of points where $\tilde{f}$ and $\mathcal{M}_{q_{0}}$ coincide: thanks to corollary 4.1. each $x+y \mathbb{S} \subset \mathbb{B}$ contains as many zeros of $\tilde{f}-\mathcal{M}_{q_{0}}$ as zeros of

$$
\begin{aligned}
& \left(1-f(q) \overline{q_{0}}\right) *\left(\tilde{f}(q)-\mathcal{M}_{q_{0}}(q)\right) *\left(1-q \bar{q}_{0}\right)= \\
& \left(f(q)-q_{0}\right) *\left(1-q \bar{q}_{0}\right)-\left(1-f(q) \bar{q}_{0}\right) *\left(q-q_{0}\right)= \\
& f(q) *\left[1-q \bar{q}_{0}+\bar{q}_{0} *\left(q-q_{0}\right)\right]-q_{0} *\left(1-q \bar{q}_{0}\right)-\left(q-q_{0}\right)= \\
& f(q)\left(1-\left|q_{0}\right|^{2}\right)-q\left(1-\left|q_{0}\right|^{2}\right)=[f(q)-q]\left(1-\left|q_{0}\right|^{2}\right) .
\end{aligned}
$$

Clearly, the zero set of the last function is the fixed point set of $f$.

Now let us suppose $f$ to have another fixed point $q_{1}=x_{1}+I y_{1} \neq q_{0}$. If $q_{0} \in x_{1}+y_{1} \mathbb{S}$ then the fixed point set contains the whole 2 -sphere $x_{1}+y_{1} \mathbb{S}$, and so does the zero set of $\tilde{f}-\mathcal{M}_{q_{0}}$; in particular $\left|\tilde{f}\left(x_{1}+J y_{1}\right)\right|=\left|\mathcal{M}_{q_{0}}\left(x_{1}+J y_{1}\right)\right|$ for all $J \in \mathbb{S}$. If, on the contrary, $q_{0}$ and $q_{1}$ lie in different spheres, then $\tilde{f}-\mathcal{M}_{q_{0}}$ has a zero $\tilde{q}_{1} \in x_{1}+y_{1} \mathbb{S}$ and in particular $\left|\tilde{f}\left(\tilde{q}_{1}\right)\right|=\left|\mathcal{M}_{q_{0}}\left(\tilde{q}_{1}\right)\right|$ with $\tilde{q}_{1} \neq q_{0}$. In both cases, according to the regular Schwarz-Pick lemma (theorem 3.7), $f$ must be a regular Möbius transformation of $\mathbb{B}$.

We are left with proving that a regular Möbius transformation of $\mathbb{B}$ having more than one fixed point in $\mathbb{B}$ must be the identity function. According to corollary 4.1 for all $a \in \mathbb{B}, u \in \partial \mathbb{B}$, the difference $(1-q \bar{a})^{-*} *(q-a) u-q$ has more than one zero in $\mathbb{B}$ if, and only if,

$$
P(q)=(q-a) u-(1-q \bar{a}) * q=q^{2} \bar{a}+q(u-1)-a u
$$

does. Now, if the last polynomial factorizes as $P(q)=(q-\alpha) *(q-\beta) \bar{a}$ then $\alpha \beta \bar{a}=-a u$. Either $a=0$ (in which case $P \equiv 0$ and the transformation coincides 
with the identity) or $|\alpha \beta|=1$. In the latter case, $\alpha$ and $\beta$ cannot both lie in $\mathbb{B}$, and $P(q)$ cannot have more than one zero in $\mathbb{B}$. Thus, $(1-q \bar{a})^{-*} *(q-a) u$ cannot have more than one fixed point in $\mathbb{B}$, and our proof is complete.

As a byproduct of the previous proof, we observe that a regular Möbius transformation of $\mathbb{B}$ having a fixed point in $\mathbb{B}$ either is the identity or has no other fixed point in $\overline{\mathbb{B}}$.

Another nice application of our main theorem 3.7 is a direct proof of a result of 13: the analog of Cartan's rigidity theorem for regular functions. In that paper, the result was proven using a "slicewise" technique: that is, reducing to the complex Cartan theorem. A direct approach is now possible, and it allows a generalization of the statement.

Theorem 4.3. Let $f: \mathbb{B} \rightarrow \mathbb{B}$ be a regular function and suppose $f$ to have a fixed point $q_{0} \in \mathbb{B}$. The following facts are equivalent:

(1) $f$ coincides with the identity function;

(2) the real differential of $f$ at $q_{0}$ is the identity;

(3) the Cullen derivative $\partial_{c} f\left(q_{0}\right)$ equals 1 ;

(4) the spherical derivative $\partial_{s} f\left(q_{0}\right)$ equals 1 ;

(5) $R_{q_{0}} f(q)$ equals $\left(1-\bar{q}_{0} * q\right)^{-*} *\left(1-\bar{q}_{0} * f(q)\right)$ at some $q \in \mathbb{B}$.

The new proof is based on a technical lemma.

Lemma 4.4. Let $f: \mathbb{B} \rightarrow \mathbb{B}$ be a regular function and let $q_{0} \in \mathbb{B}$. If $R_{q_{0}} f(q)$ equals the quotient

$$
\left(1-\bar{q}_{0} * q\right)^{-*} *\left(1-\overline{f\left(q_{0}\right)} * f(q)\right)
$$

at any point of $\mathbb{B}$, then $f$ is a Möbius transformation of $\mathbb{B}$.

Proof. By theorem 3.7 .

$$
\left|R_{q_{0}} f(q) *\left(1-\overline{f\left(q_{0}\right)} * f(q)\right)^{-*}\right| \leq\left|\left(1-\bar{q}_{0} * q\right)^{-*}\right|
$$

and $f$ is a regular Möbius transformation if equality holds at any point of $\mathbb{B}$. This is true, in particular, if

$$
R_{q_{0}} f(q) *\left(1-\overline{f\left(q_{0}\right)} * f(q)\right)^{-*}-\left(1-\bar{q}_{0} * q\right)^{-*}
$$

vanishes at any point of $\mathbb{B}$. This is equivalent to the vanishing of

$$
R_{q_{0}} f(q)-\left(1-\bar{q}_{0} * q\right)^{-*} *\left(1-\overline{f\left(q_{0}\right)} * f(q)\right)
$$

at some $q \in \mathbb{B}$. 
Proof of theorem 4.3. (1) $\Rightarrow(2) \Rightarrow(3)$ : these implications are obvious. $(3) \Rightarrow(5)$ : we already know that $R_{q_{0}} f\left(q_{0}\right)=\partial_{c} f\left(q_{0}\right)$. Moreover if $f\left(q_{0}\right)=q_{0}$ then the quotient

$$
Q(q)=\left(1-q \bar{q}_{0}\right)^{-*} *\left(1-\bar{q}_{0} * f(q)\right)
$$

equals 1 at $q_{0}$, thanks to the fact that $\bar{q}_{0} * f(q)=\bar{q}_{0} f\left(\bar{q}_{0}^{-1} q \bar{q}_{0}\right)$ (by theorem 2.5). $(5) \Rightarrow(1)$ : in the case of a fixed point $q_{0}$, quotient (32) equals the aforementioned $Q(q)$. According to the previous lemma, if $Q(q)$ equals $R_{q_{0}} f(q)$ at any point of $\mathbb{B}$ then $f$ is a regular Möbius transformation. We are left with proving that if $f$ is a regular Möbius transformation $f(q)=(1-q \bar{a})^{-*} *(q-a) u$ and if

$$
\begin{aligned}
& R_{q_{0}} f(q)-Q(q)= \\
& {\left[\left(q-q_{0}\right)^{-*}+\left(1-q \bar{q}_{0}\right)^{-*} \bar{q}_{0}\right] * f(q)-\left(q-q_{0}\right)^{-*} q_{0}-\left(1-q \bar{q}_{0}\right)^{-*}=} \\
& \left(q-q_{0}\right)^{-*} *\left(1-q \bar{q}_{0}\right)^{-*} *\left\{\left[1-q \bar{q}_{0}+\left(q-q_{0}\right) \bar{q}_{0}\right] * f(q)-\left(1-q \bar{q}_{0}\right) q_{0}-\left(q-q_{0}\right)\right\}= \\
& \left(q-q_{0}\right)^{-*} *\left(1-q \bar{q}_{0}\right)^{-*} *\left(1-\left|q_{0}\right|^{2}\right) *[f(q)-q]
\end{aligned}
$$

has a zero in $\mathbb{B}$ then $f$ is identity. The latter condition is equivalent to the existence of a zero $q_{1} \in \mathbb{B}$ for

$$
\left(1-q \bar{q}_{0}\right) *\left[R_{q_{0}} f(q)-Q(q)\right]\left(1-\left|q_{0}\right|^{2}\right)^{-1}=\left(q-q_{0}\right)^{-*} *[f(q)-q],
$$

i.e., to the existence of a regular $g: \mathbb{B} \rightarrow \mathbb{H}$ such that

$$
f(q)-q=\left(q-q_{0}\right) *\left(q-q_{1}\right) * g(q) .
$$

We have already seen in the proof of theorem 4.2 that $f(q)-q=(1-q \bar{a})^{-*} *$ $(q-\alpha) *(q-\beta) \bar{a}$ where $\alpha, \beta$ cannot both lie in $\mathbb{B}$. These two facts are only compatible if $\bar{a}=0$ and $g \equiv 0$, that is, if $f=i d$.

$(1) \Leftrightarrow(4)$ : when $f\left(q_{0}\right)=q_{0}$ then the spherical derivative

$$
\partial_{s} f\left(q_{0}\right)=\left(q_{0}-\bar{q}_{0}\right)^{-1}\left(q_{0}-f\left(\bar{q}_{0}\right)\right)
$$

equals 1 if, and only if, $\bar{q}_{0}$ is a fixed point of $f$, too. According to theorem 4.2 the latter is equivalent to $f=i d$.

For the sake of completeness, we conclude this section identifying explicitly which regular Möbius transformations fix a point $q_{0} \in \mathbb{B}$. Let us denote

$$
S^{3}=\left\{\left[\begin{array}{ll}
v & 0 \\
0 & 1
\end{array}\right]: v \in \partial \mathbb{B}\right\} \leq S p(1,1)
$$

Proposition 4.5. For each $q_{0} \in \mathbb{B}$, the class of regular Möbius transformations fixing $q_{0}$ is the orbit of the identity function under the right action of the subgroup

$$
C\left(q_{0}\right) \cdot S^{3} \cdot C\left(q_{0}\right)^{-1} \leq S p(1,1)
$$


with $C\left(q_{0}\right)=\left[\begin{array}{cc}1 & -\bar{q}_{0} \\ -q_{0} & 1\end{array}\right]$. In other words, $(1-q \bar{a})^{-*} *(q-a) u$ fixes $q_{0}$ if, and only if,

$$
\begin{aligned}
u & =\left(1-q_{0} v \bar{q}_{0}\right)^{-1}\left(v-\left|q_{0}\right|^{2}\right) \\
a & =q_{0}(1-\bar{v})\left(1-q_{0} \bar{v} \bar{q}_{0}\right)^{-1}
\end{aligned}
$$

for some $v \in \partial \mathbb{B}$.

Proof. If $f$ is a regular Möbius transformation fixing $q_{0}$ then $\tilde{f}=f . C\left(q_{0}\right)$ is a regular Möbius transformation mapping $q_{0}$ to 0 . Hence $\tilde{f}(q)=\left(1-q \bar{q}_{0}\right)^{-*} *$ $\left(q-q_{0}\right) v$ for some $v \in \partial \mathbb{B}$. In other words,

$$
f \cdot C\left(q_{0}\right)=i d \cdot C\left(q_{0}\right) \cdot\left[\begin{array}{ll}
v & 0 \\
0 & 1
\end{array}\right]
$$

or, equivalently, $f=i d$. $A$ for some $A \in C\left(q_{0}\right) \cdot S^{3} \cdot C\left(q_{0}\right)^{-1}$. The final statement follows by direct computation, forcing

$$
\left[\begin{array}{cc}
1 & -\bar{q}_{0} \\
-q_{0} & 1
\end{array}\right]\left[\begin{array}{ll}
v & 0 \\
0 & 1
\end{array}\right]\left[\begin{array}{cc}
1 & -\bar{q}_{0} \\
-q_{0} & 1
\end{array}\right]^{-1}
$$

and

$$
\left[\begin{array}{cc}
1 & -\bar{a} \\
-a & 1
\end{array}\right]\left[\begin{array}{cc}
u & 0 \\
0 & 1
\end{array}\right]
$$

to induce the same transformation.

\section{$5 \quad$ Higher order estimates}

As in the complex case, the quaternionic Schwarz-Pick lemma admits higher order generalizations. Let us denote the $n$th Cullen derivative of a regular function $f$ as $\partial_{c}^{n} f$. Let $\left(q-q_{0}\right)^{* n}=\left(q-q_{0}\right) * \ldots *\left(q-q_{0}\right)$ denote the $*$-product of $n$ copies of $q \mapsto q-q_{0}$. The next theorem was proven in [10].

Theorem 5.1. Let $\Omega$ be a domain in $\mathbb{H}$. A function $f: \Omega \rightarrow \mathbb{H}$ is regular if and only if, for each $q_{0} \in \Omega$

$$
f(q)=\sum_{n \in \mathbb{N}}\left(q-q_{0}\right)^{* n} \frac{\partial_{c}^{n} f\left(q_{0}\right)}{n !}
$$

in a ball centered at $q_{0}$ with respect to the non-Euclidean distance

$$
\sigma(q, p)= \begin{cases}|q-p| & \text { if } p, q \text { lie on the same complex plane } L_{I} \\ \omega(q, p) & \text { otherwise }\end{cases}
$$

where

$$
\omega(q, p)=\sqrt{[\operatorname{Re}(q)-\operatorname{Re}(p)]^{2}+[|\operatorname{Im}(q)|+|\operatorname{Im}(p)|]^{2}} .
$$


Theorem 3.7 extends to the next result.

Theorem 5.2. Let $f: \mathbb{B} \rightarrow \mathbb{B}$ be a regular function, let $q_{0} \in \mathbb{B}$. If $\partial_{c}^{m} f\left(q_{0}\right)=0$ for $1 \leq m \leq n-1$ then

$$
\left|\left(f(q)-f\left(q_{0}\right)\right) *\left(1-\overline{f\left(q_{0}\right)} * f(q)\right)^{-*}\right| \leq\left|\left(q-q_{0}\right)^{* n} *\left(1-\bar{q}_{0} * q\right)^{-* n}\right|
$$

for $q \in \mathbb{B}$. Furthermore,

$$
\left|\partial_{c}^{n} f *\left(1-\overline{f\left(q_{0}\right)} * f\right)^{-*}\right|_{\left.\right|_{q_{0}}} \leq \frac{n !}{\left(1-\left|q_{0}\right|^{2}\right)^{n}}
$$

We point out that $\frac{n !}{\left(1-\left|q_{0}\right|^{2}\right)^{n}}$ is the $n$th Cullen derivative of

$$
\left(q-q_{0}\right)^{* n} *\left(1-\bar{q}_{0} * q\right)^{-* n}=\mathcal{M}_{q_{0}}^{* n}(q) .
$$

Proof. If $\partial_{c}^{m} f\left(q_{0}\right)=0$ for $1 \leq m \leq n-1$ then setting $q_{1}=f\left(q_{0}\right)$ and $\tilde{f}=$ $\left(f-q_{1}\right) *\left(1-\bar{q}_{1} * f\right)^{-*}$ defines a regular $\tilde{f}: \mathbb{B} \rightarrow \mathbb{B}$ with

$$
\partial_{c}^{m} \tilde{f}=\sum_{k=0}^{m-1} \partial_{c}^{m-k} f * \partial_{c}^{k}\left(1-\bar{q}_{1} * f\right)^{-*}\left(\begin{array}{c}
m \\
k
\end{array}\right)+\left(f-q_{1}\right) * \partial_{c}^{m}\left(1-\bar{q}_{1} * f\right)^{-*} .
$$

Hence, $\partial_{c}^{m} \tilde{f}\left(q_{0}\right)=0$ for $0 \leq m \leq n-1$, i.e., $\tilde{f}(q)=\left(q-q_{0}\right)^{* n} * g(q)$ for some regular $g: \mathbb{B} \rightarrow \mathbb{H}$. Reasoning as in theorem 3.2 we prove that $\mathcal{M}_{q_{0}}^{-* n} * \tilde{f}(q)$ is a regular function $\mathbb{B} \rightarrow \mathbb{B}$ and derive that for all $q \in \mathbb{B}$

$$
|\tilde{f}(q)| \leq\left|\mathcal{M}_{q_{0}}^{* n}(q)\right|
$$

and

$$
\left|\left(q-q_{0}\right)^{-* n} * \tilde{f}(q)\right| \leq\left|\left(1-\bar{q}_{0} * q\right)^{-* n}\right| .
$$

The latter implies

$$
\left|\partial_{c}^{n} \tilde{f}\left(q_{0}\right)\right| \leq \frac{n !}{\left(1-\left|q_{0}\right|^{2}\right)^{n}}
$$

and observing

$$
\partial_{c}^{n} \tilde{f}\left(q_{0}\right)=\left[\partial_{c}^{n} f *\left(1-\bar{q}_{1} * f\right)^{-*}\right]_{\left.\right|_{q_{0}}}
$$

completes the proof.

Finally, we generalize theorem 3.7 in a different direction. We recall that the spherical derivative $\partial_{s} f$ is constant on each sphere $x_{0}+y_{0} \mathbb{S}$ and that it is not regular unless it is constant. Hence, iterated spherical derivation is meaningless. However, it makes sense to iterate the operator $R_{q_{0}}$ defined by formula (8). This led in 22] to the following result, where we use the notation

$$
U\left(x_{0}+y_{0} \mathbb{S}, r\right)=\left\{q \in \mathbb{H}||\left(q-x_{0}\right)^{2}+y_{0}^{2} \mid<r^{2}\right\}
$$

for all $x_{0}, y_{0} \in \mathbb{R}, r>0$, and we denote the composition of $R_{\bar{q}_{0}}$ and $R_{q_{0}}$ by juxtaposition and the $n$th iterate of $R_{\bar{q}_{0}} R_{q_{0}}$ by $\left(R_{\bar{q}_{0}} R_{q_{0}}\right)^{n}$. 
Theorem 5.3. Let $f$ be a regular function on $\Omega=B(0, R)$, and let $U\left(x_{0}+\right.$ $\left.y_{0} \mathbb{S}, r\right) \subseteq \Omega$. Then for each $q_{0} \in x_{0}+y_{0} \mathbb{S}$ there exists $\left\{A_{n}\right\}_{n \in \mathbb{N}} \subset \mathbb{H}$ such that

$$
f(q)=\sum_{n \in \mathbb{N}}\left[\left(q-x_{0}\right)^{2}+y_{0}^{2}\right]^{n}\left[A_{2 n}+\left(q-q_{0}\right) A_{2 n+1}\right]
$$

for all $q \in U\left(x_{0}+y_{0} \mathbb{S}, r\right)$. Namely, $A_{2 n}=\left(R_{\bar{q}_{0}} R_{q_{0}}\right)^{n} f\left(q_{0}\right)$ and $A_{2 n+1}=$ $R_{q_{0}}\left(R_{\bar{q}_{0}} R_{q_{0}}\right)^{n} f\left(\bar{q}_{0}\right)$ for all $n \in \mathbb{N}$.

We are now ready for the announced higher order estimates. We recall that $g^{s}$ denotes the function obtained from $g$ by symmetrization (see definition 2.2.).

Theorem 5.4. Let $f: \mathbb{B} \rightarrow \mathbb{B}$ be a regular function and let $q_{0} \in \mathbb{B}$. If the coefficients $A_{m}$ of the expansion (41) vanish for $1 \leq m \leq 2 n-1$ then

$$
\begin{aligned}
\left|\left(f(q)-f\left(q_{0}\right)\right) *\left(1-\overline{f\left(q_{0}\right)} * f(q)\right)^{-*}\right| & \leq\left|\mathcal{M}_{q_{0}}^{s}(q)\right|^{n} \\
\left|\left(R_{\bar{q}_{0}} R_{q_{0}}\right)^{n} f(q) *\left(1-\overline{f\left(q_{0}\right)} * f(q)\right)^{-*}\right| & \leq\left|\left(1-q x_{0}\right)^{2}+\left(q y_{0}\right)^{2}\right|^{-n}
\end{aligned}
$$

for $q \in \mathbb{B}$. If $A_{2 n}=0$ as well then

$$
\begin{aligned}
\left|\left(f(q)-f\left(q_{0}\right)\right) *\left(1-\overline{f\left(q_{0}\right)} * f(q)\right)^{-*}\right| & \leq\left|\mathcal{M}_{q_{0}}^{s}(q)\right|^{n}\left|\mathcal{M}_{q_{0}}(q)\right| \\
\left|R_{q_{0}}\left(R_{\bar{q}_{0}} R_{q_{0}}\right)^{n} f(q) *\left(1-\overline{f\left(q_{0}\right)} * f(q)\right)^{-*}\right| & \leq\left|\left(1-q x_{0}\right)^{2}+\left(q y_{0}\right)^{2}\right|^{-n} \cdot\left|\left(1-q \bar{q}_{0}\right)^{-*}\right|
\end{aligned}
$$

Proof. Let us set $\tilde{f}(q)=\left(f(q)-f\left(q_{0}\right)\right) *\left(1-\overline{f\left(q_{0}\right)} * f(q)\right)^{-*}$. This defines a regular $\tilde{f}: \mathbb{B} \rightarrow \mathbb{B}$ with $\tilde{f}\left(q_{0}\right)=0$ and

$$
R_{q_{0}} \tilde{f}(q)=\left(q-q_{0}\right)^{-*} * \tilde{f}(q)=R_{q_{0}} f(q) *\left(1-\overline{f\left(q_{0}\right)} * f(q)\right)^{-*} .
$$

Thanks to the hypothesis $A_{1}=R_{q_{0}} f\left(\bar{q}_{0}\right)=0$, we conclude that $R_{q_{0}} \tilde{f}\left(\bar{q}_{0}\right)=0$ so that

$$
\begin{aligned}
R_{\bar{q}_{0}} R_{q_{0}} \tilde{f}(q) & =\left(q-\bar{q}_{0}\right)^{-*} * R_{q_{0}} \tilde{f}(q)=\left[\left(q-x_{0}\right)^{2}+y_{0}^{2}\right]^{-1} \tilde{f}(q) \\
& =R_{\bar{q}_{0}} R_{q_{0}} f(q) *\left(1-\overline{f\left(q_{0}\right)} * f(q)\right)^{-*} .
\end{aligned}
$$

Iterating this process, we conclude that

$$
\begin{aligned}
R_{q_{0}}\left(R_{\bar{q}_{0}} R_{q_{0}}\right)^{k-1} \tilde{f}(q) & =\left[\left(q-x_{0}\right)^{2}+y_{0}^{2}\right]^{-k+1}\left(q-q_{0}\right)^{-*} * \tilde{f}(q) \\
& =R_{q_{0}}\left(R_{\bar{q}_{0}} R_{q_{0}}\right)^{k-1} f(q) *\left(1-\overline{f\left(q_{0}\right)} * f(q)\right)^{-*}, \\
\left(R_{\bar{q}_{0}} R_{q_{0}}\right)^{k} \tilde{f}(q) & =\left[\left(q-x_{0}\right)^{2}+y_{0}^{2}\right]^{-k} \tilde{f}(q) \\
& =\left(R_{\bar{q}_{0}} R_{q_{0}}\right)^{k} f(q) *\left(1-\overline{f\left(q_{0}\right)} * f(q)\right)^{-*}
\end{aligned}
$$

for all $1 \leq k \leq n$, and that the coefficients $\widetilde{A}_{m}$ of the expansion of $\tilde{f}$ vanish for all $0 \leq m \leq 2 n-1$. As a consequence, $\tilde{f}(q)=\left[\left(q-x_{0}\right)^{2}+y_{0}^{2}\right]^{n} g(q)$ for some regular function $g$. Let us consider

$$
\mathcal{M}_{q_{0}}^{s}(q)=\left(1-q \bar{q}_{0}\right)^{-s}\left(q-q_{0}\right)^{s}=\left[\left(1-q x_{0}\right)^{2}+\left(q y_{0}\right)^{2}\right]^{-1}\left[\left(q-x_{0}\right)^{2}+y_{0}^{2}\right]
$$


and its $n$th power $\left(\mathcal{M}_{q_{0}}^{s}\right)^{n}$ : then

$$
\left(\mathcal{M}_{q_{0}}^{s}(q)\right)^{-n} \tilde{f}(q)=\left[\left(1-q x_{0}\right)^{2}+\left(q y_{0}\right)^{2}\right]^{n} g(q)
$$

is a regular function $h$ on $\mathbb{B}$. Now, $|h|=\left|\mathcal{M}_{q_{0}}^{s}(q)\right|^{-n}|\tilde{f}(q)| \leq\left|\mathcal{M}_{q_{0}}^{s}(q)\right|^{-n}$ where $\mathcal{M}_{q_{0}}^{s}=\mathcal{M}_{q_{0}} * \mathcal{M}_{q_{0}}^{c}=\mathcal{M}_{q_{0}} * \mathcal{M}_{\bar{q}_{0}}$ maps $\partial \mathbb{B}$ to $\partial \mathbb{B}$ by lemma 3.3. Reasoning as in theorem 3.2 we can prove that $|h| \leq 1$ and equations (42) follow by direct computation.

Finally, if $A_{2 n}=0$ then $\widetilde{A}_{2 n}=0$ and $g(q)=\left[\left(q-x_{0}\right)^{2}+y_{0}^{2}\right]^{-n} \tilde{f}(q)$ has a zero at $q_{0}$. Thus, $h(q)=\left[\left(1-q x_{0}\right)^{2}+\left(q y_{0}\right)^{2}\right]^{n} g(q)$ is a regular function $\mathbb{B} \rightarrow \mathbb{B}$ having a zero at $q_{0}$. By theorem [3.7, $|h| \leq\left|\mathcal{M}_{q_{0}}\right|$ and equations (43) follow (making use of lemma 3.3).

We believe that the two results proven in this section, however technical their statements may appear, show that the quaternionic Schwarz-Pick lemma establishes a strong link between the differential and multiplicative properties of the regular self-maps of $\mathbb{B}$. This recalls the complex setting, but in a many-sided way that reflects the richness of the non-commutative context.

\section{References}

[1] M. Abate. Iteration theory of holomorphic maps on taut manifolds. Research and Lecture Notes in Mathematics. Complex Analysis and Geometry. Mediterranean Press, Rende, 1989.

[2] L. V. Ahlfors. Möbius transformations in $\mathbf{R}^{n}$ expressed through $2 \times 2$ matrices of Clifford numbers. Complex Variables Theory Appl., 5(2-4):215$224,1986$.

[3] C. Bisi and G. Gentili. Möbius transformations and the Poincaré distance in the quaternionic setting. Indiana Univ. Math. J., 58(6):2729-2764, 2009.

[4] C. Bisi and C. Stoppato. Regular vs. classical Möbius transformations of the quaternionic unit ball. In G. Gentili, I. Sabadini, M. V. Shapiro, F. Sommen, and D. C. Struppa, editors, Advances in Hypercomplex Analysis, Springer INdAM Series. Springer, Milan, 2013.

[5] F. Brackx, R. Delanghe, and F. Sommen. Clifford analysis, volume 76 of Research Notes in Mathematics. Pitman (Advanced Publishing Program), Boston, MA, 1982.

[6] F. Colombo, G. Gentili, I. Sabadini, and D. Struppa. Extension results for slice regular functions of a quaternionic variable. Adv. Math., 222(5):1793$1808,2009$.

[7] R. Fueter. Die Funktionentheorie der Differentialgleichungen $\Delta u=0$ und $\Delta \Delta u=0$ mit vier reellen Variablen. Comment. Math. Helv., 7(1):307-330, 1934. 
[8] R. Fueter. Über die analytische Darstellung der regulären Funktionen einer Quaternionenvariablen. Comment. Math. Helv., 8(1):371-378, 1935.

[9] G. Gentili and C. Stoppato. Zeros of regular functions and polynomials of a quaternionic variable. Michigan Math. J., 56(3):655-667, 2008.

[10] G. Gentili and C. Stoppato. Power series and analyticity over the quaternions. Math. Ann., 352(1):113-131, 2012.

[11] G. Gentili and D. C. Struppa. A new approach to Cullen-regular functions of a quaternionic variable. C. R. Math. Acad. Sci. Paris, 342(10):741-744, 2006 .

[12] G. Gentili and D. C. Struppa. A new theory of regular functions of a quaternionic variable. Adv. Math., 216(1):279-301, 2007.

[13] G. Gentili and F. Vlacci. Rigidity for regular functions over Hamilton and Cayley numbers and a boundary Schwarz Lemma. Indag. Math. (N.S.), 19(4):535-545, 2008.

[14] R. Ghiloni and A. Perotti. Slice regular functions on real alternative algebras. Adv. Math., 226(2):1662-1691, 2011.

[15] H. Maass. Automorphe Funktionen von meheren Veränderlichen und Dirichletsche Reihen. Abh. Math. Sem. Univ. Hamburg, 16:72-100, 1949.

[16] A. Perotti. Regular quaternionic functions and conformal mappings. Cubo, 11(1):123-143, 2009.

[17] G. Pick. Über die Beschränkungen analytischer Funktionen, welche durch vorgegebene Funktionswerte bewirkt werden. Math. Ann., 77(1):7-23, 1915 .

[18] G. Pick. Über eine Eigenschaft der konformen Abbildung kreisförmiger Bereiche. Math. Ann., 77(1):1-6, 1915.

[19] L. H. Rowen. Ring theory. Academic Press Inc., Boston, MA, student edition, 1991.

[20] C. Stoppato. Poles of regular quaternionic functions. Complex Var. Elliptic Equ., 54(11):1001-1018, 2009.

[21] C. Stoppato. Regular Moebius transformations of the space of quaternions. Ann. Global Anal. Geom., 39(4):387-401, 2011.

[22] C. Stoppato. A new series expansion for slice regular functions. Adv. Math., 231(3-4):1401-1416, 2012.

[23] A. Sudbery. Quaternionic analysis. Math. Proc. Cambridge Philos. Soc., 85(2):199-224, 1979. 
[24] K. T. Vahlen. Ueber Bewegungen und complexe Zahlen. Math. Ann., 55(4):585-593, 1902.

[25] E. Vesentini. Capitoli scelti della teoria delle funzioni olomorfe. Unione Matematica Italiana, Bologna, 1984.

[26] Y. Yang and T. Qian. Schwarz lemma in Euclidean spaces. Complex Var. Elliptic Equ., 51(7):653-659, 2006. 